\title{
Small bowel adenocarcinoma in Lynch syndrome: A case report
}

\author{
KE-KANG SUN, GANG LIU, XIAOJUN SHEN and XIAOYANG WU \\ Department of Gastrointestinal Surgery, Kunshan First People's Hospital Affiliated to Jiangsu University, \\ Kunshan, Jiangsu 215300, P.R. China
}

Received April 7, 2015; Accepted May 23, 2016

DOI: $10.3892 / \mathrm{ol} .2016 .4753$

\begin{abstract}
Small bowel adenocarcinoma is part of the tumor spectrum of Lynch syndrome, which is caused by germline mutations in the mismatch repair genes. The present study describes the case of a 51-year-old man fulfilling the Amsterdam II criteria for Lynch syndrome, who had a 15-mm early-stage colorectal cancer resected endoscopically from the ascending colon. Due to upper abdominal discomfort after eating and consequent anorexia, a computed tomography scan performed 1 month later showed a tumoral mass of the upper jejunum with local lymphadenopathy. The laparotomy revealed a completely obstructing mass. Intraoperative frozen section showed a small bowel adenocarcinoma. Subsequent genetic testing confirmed the germline mutation of mutL homolog 1 . The patient received 6 cycles of an adjuvant folinic acid, fluorouracil and ocaliplatin chemotherapy regimen. The latest CT scan, 16 months after the chemotherapy, did not show any recurrence. This case highlights the importance of considering the possibility of small bowel adenocarcinoma in patients with upper bowel obstruction, particularly for patients with Lynch syndrome.
\end{abstract}

\section{Introduction}

Lynch syndrome or hereditary non-polyposis colorectal cancer, is an autosomal dominant condition that predisposes individuals to multiple cancer types and is caused by germline mutations in one of the mismatch repair genes, usually (mutL homolog 1 (MLH1), or mutS homolog (MSH)2 or MSH6 (1-3). Affected individuals are highly susceptible to colorectal and endometrial cancer, but also to cancers of the stomach, ovary, urinary tract, hepatobiliary tract, pancreas, and small bowel. In Lynch syndrome, the risk of developing small bowel cancer within a lifetime is $\sim 4 \%$ (4), which is almost identical to the risk of developing colorectal cancer for individuals in the

Correspondence to: $\mathrm{Mr}$. Xiaoyang $\mathrm{Wu}$, Department of Gastrointestinal Surgery, Kunshan First People's Hospital Affiliated to Jiangsu University, 2nd Floor, Building 2, 91 Qianjin Road, Kunshan, Jiangsu 215300, P.R. China

E-mail: fsyx7skk@163.com

Key words: small bowel adenocarcinoma, Lynch syndrome general population (5). A lack of effective screening methods and the relative paucity of presenting symptoms contribute to a high percentage of cases at the advanced stage at the time of diagnosis in small bowel cancer (6). Small bowel adenocarcinoma has a poor prognosis at all stages, with a 5-year overall survival rate ranging from $14-33 \%(7)$. The present study describes the case of a patient with small bowel adenocarcinoma and Lynch syndrome, in whom ascending colon cancer was diagnosed 1 month earlier. This case highlights the requirement to consider the extracolonic manifestations of Lynch syndrome, particularly in patients with upper bowel obstruction, abdominal pain or occult gastrointestinal bleeding.

\section{Case report}

A 51-year-old man was admitted to Department of Gastroenterology, Kunshan First People's Hospital Affiliated to Jiangsu University (Kunshan, Jiangsu, China) on September 16, 2014, due to upper abdominal discomfort after eating and consequent anorexia that had persisted for 2 weeks. The patient's past medical history included an endoscopic resection of a 15-mm early-stage colorectal cancer from the ascending colon on July 30, 2014. A stool test was positive for fecal occult blood. An esophagogastroduodenoscopy and colonoscopy were unremarkable. A subsequent contrast-enhanced abdominal computed tomography (CT) scan was required, which demonstrated an obstructing mass in the proximal jejunum leading to expansion of the duodenum (Fig. 1). Upon review of the abdominal CT scan that had been performed at the time of the endoscopic resection 1 month previously, the mucosal thickening of the small intestine was found to have already existed, although it was not so marked (Fig. 2).

A laparotomy was performed, resulting in the resection of a small bowel mass and its surrounding tissue with the primary anastomosis. The surgery was without complication and post-operative management was uneventful. The histology report demonstrated a moderately-differentiated adenocarcinoma partially comprised of a poorly-differentiated region. The microscopic findings showed cell clusters and cords with marked tissue structure atypia and invasion of the the visceral peritoneum, and metastases of 5/18 lymph nodes. The definitive diagnosis was primary adenocarcinoma of the duodenal-jejunal junction, T4N1M0, stage III according to the Tumor-Node-Metastasis classification of malignant tumors (American Joint Committee on Cancer) $(8,9)$. Subsequent 


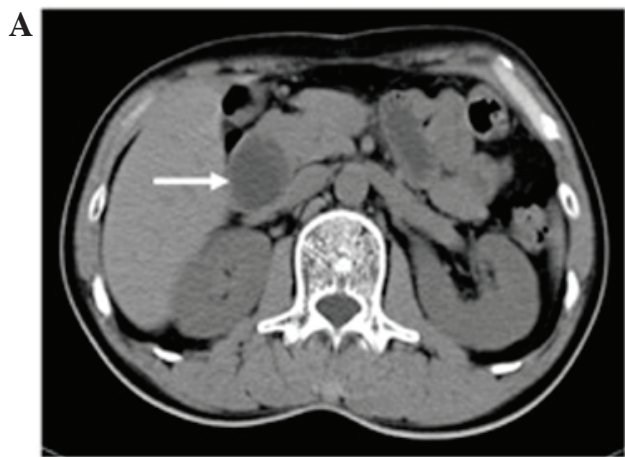

$\mathbf{B}$
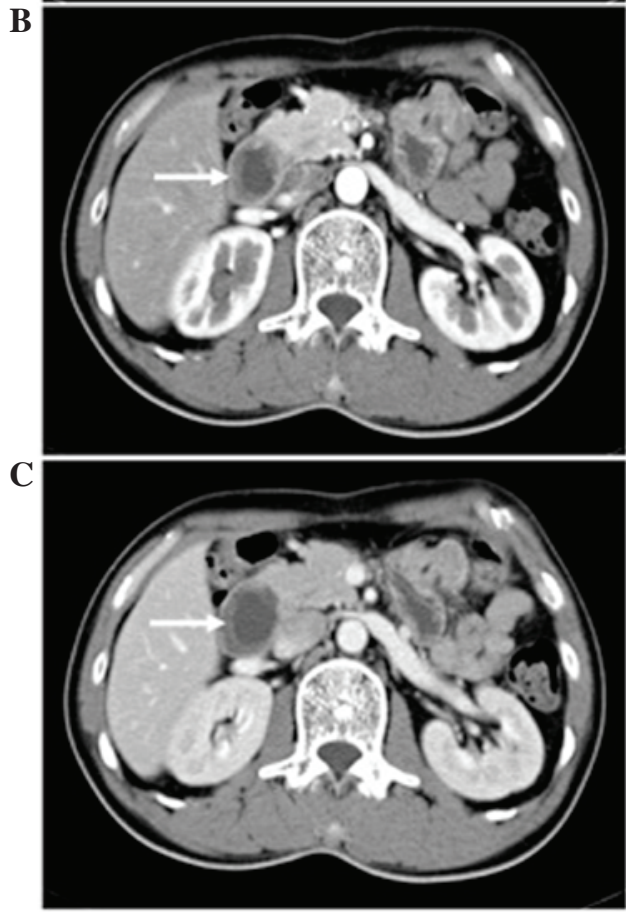

Figure 1. Abdominal computed tomography (July 27, 2014) showing an (A) unenhanced view, and (B) arterial and (C) portal venous phase images of the obstructing mass in the proximal jejunum leading to expansion of the duodenum.

genetic testing confirmed the germline mutation of MLH1. DNA was extracted by the use of a High Pure Polymerase Chain Recation (PCR) Template Purification kit (Roche Diagnostics, Mannheim, Germany). DNA isolation from whole blood of the patient and their relatives DNA was extracted with the use of QIAamp DNA Blood Midi or Mini kits (Qiagen, Hilden, Germany), according to the manufacturer's instructions. All coding exons of the MLH1 gene were amplified by polymerase chain reaction. PCR products were purified and sequenced in both directions. Sequencing reactions were performed using BigDye terminator cycle sequencing kit v.3.1 (Applied Biosystems, Foster City, CA, USA). The products of the sequencing reactions were run and analyzed using an ABIPRISM 3130 Genetic Analyzer (Perkin Elmer, Boston, MA, USA). Large deletion detection multiple ligation-dependent probe amplification (MLPA) genomic deletions were tested using SALSA MLPA kit P003 MLH/MSH2 (MRC-Holland BV, Amsterdam, Netherlands) according to the manufacturer's instructions. The amplification products were analyzed by capillary gel electrophoresis (ABI-3130). Deletion of one copy
A

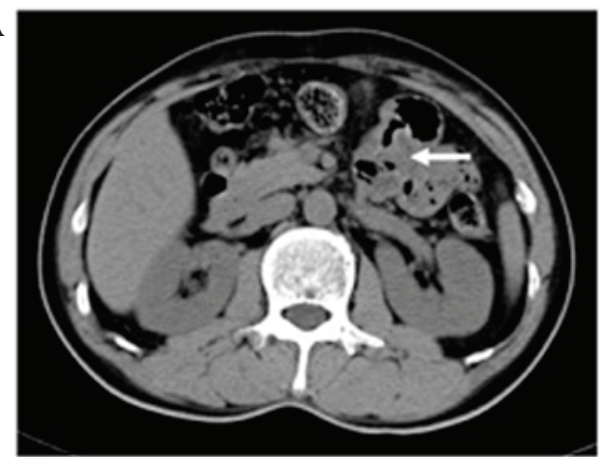

$\mathbf{B}$

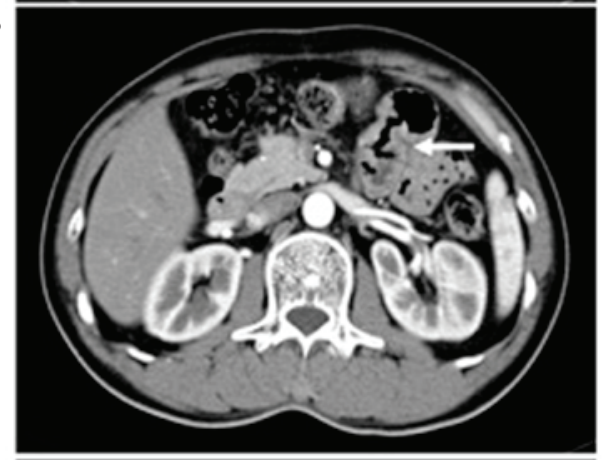

C

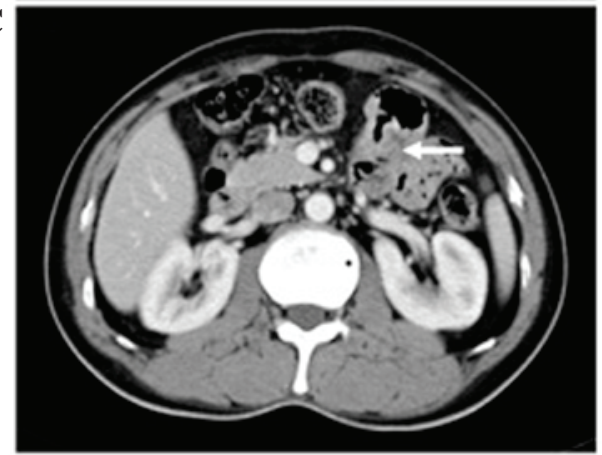

Figure 2. Abdominal computed tomography (September 23, 2014) showing an (A) unenhanced view, and (B) arterial and (C) portal venous phase images of the mucosal thickening of the upper jejunum.

of a probe targeted sequence was stated if the relative peak area for that probe amplification product had been reduced by $35-55 \%$ compared with a negative control sample.

Genetic testing had no effect on the follow-up treatment, and there was no associated targeted drug on the market. The patient therefore received 6 cycles of an adjuvant folinic acid, fluorouracil and ocaliplatin chemotherapy regimen. The latest CT scan, 16 months after the chemotherapy, did not show any recurrence.

Combined with patient medical history, these results meant that the patient was diagnosed with small bowel carcinoma, revealing Lynch syndrome.

\section{Discussion}

Small bowel adenocarcinomas are rare and account for $2 \%$ of all gastrointestinal tumors (10). The incidence of small bowel adenocarcinomas is more frequent in certain hereditary syndromes, including familial adenomatous polyposis, Peutz-Jeghers syndrome and Lynch syndrome (11). Small bowel cancer is the first malignant manifestation of Lynch 
syndrome, and it generally occurs 10-20 years earlier in these patients compared with individuals in the general population $(11,12)$. In Lynch syndrome, the risk of developing small bowel cancer within a lifetime is almost identical to the risk of developing colorectal cancer for individuals in the general population $(4,13)$. In the case of Lynch syndrome, the risk of developing small bowel adenocarcinoma has been estimated at $1-4 \%$ (14). Small bowel adenocarcinoma has a poor prognosis, with the 5-year overall survival rate not exceeding 30\% (15). Clinical symptoms are frequently non-specific, which explains a late diagnosis at stages T3 and T4 in the majority of cases. The present case highlights the requirement to consider the extracolonic manifestations of Lynch syndrome, particularly in patients with upper bowel obstruction, abdominal pain or occult gastrointestinal bleeding. Screening for small bowel cancer is generally recommended (4).

Small bowel tumors are often not visualized by both esophagogastroduodenoscopy and colonoscopy in the first instance, and further investigations to visualise the small bowel are crucial, generally in the form of magnetic resonance enterography, CT colonography or wireless capsule endoscopy, which have all been shown to be effective in reaching the diagnosis (16). The treatment of small bowel adenocarcinoma remains centered around radical surgical resection (6). A complete resection of the disease is one of the most important prognostic factors for a patient's survival, and adjuvant chemotherapy is also necessary (2). Previous studies have suggested that chemotherapy is common and beneficial in small bowel adenocarcinoma (17), but the optimal chemotherapy regimen and the degree of benefit remain to be defined.

In conclusion, small bowel adenocarcinoma can be the first and the only manifestation of Lynch syndrome. A sound approach to investigate the difference between small bowel adenocarcinoma in individuals with Lynch syndrome and small bowel adenocarcinoma in the general population is urgently required. Patients with Lynch syndrome generally present with small bowel cancer 10-20 years earlier than the general population, and small bowel cancer may be the first clinical manifestation of Lynch syndrome. The present study describes the case of a patient with small bowel adenocarcinoma and Lynch syndrome, in whom ascending colon cancer was diagnosed 1 month earlier. In view of the improved accessibility of the small bowel with the introduction of capsule endoscopy and double balloon enteroscopy, the question of whether patients should be screened for small bowel neoplasia has regained attention.

\section{References}

1. Coco D, Leanza S and Boccoli G: Small bowel adenocarcinoma (SBA) three years after colonic adenocarcinoma in an elderly patient: Case report in a National Institute of Health and Aging (INRCA) and review of the literature. Int J Surg Case Rep 5: 939-943, 2014

2. Chaaya A and Heller SJ: Introduction to small bowel tumors. Tech Gastrointest Endosc 14: 88-93, 2012.

3. Tanyi M, Olasz J, Tanyi JL, Tóth L, Antal-Szalmás P, Ress Z, Bubán T, Palatka K, András C, Urbancsek H, et al: MLH1 and MSH2 mutation screening in HNPCC families of Hungary-Two new MMR gene mutations. Eur J Surg Oncol 40: 1445-1452, 2014.

4. Koornstra JJ, Kleibeuker JH and Vasen HF: Small-bowel cancer in Lynch syndrome: Is it time for surveillance? Lancet Oncol 9: 901-905, 2008.

5. Marmo R, Rotondano G, Riccio G, D'Angella R, Rescinito M, Rescinito A, Bianco MA and Cipolletta L: Small-bowel adenocarcinoma diagnosed via capsule endoscopy in a patient found to have hereditary nonpolyposis colorectal cancer. Gastrointest Endosc 65: 524-525, 2007.

6. Delaunoit T, Neczyporenko F, Limburg PJ and Erlichman C: Small bowel adenocarcinoma: A rare but aggressive disease. Clin Colorectal Cancer 4: 241-251, 2004.

7. Bilimoria KY, Bentrem DJ, Wayne JD, Ko CY, Bennett CL, Talamonti MS: Small bowel cancer in the United States: Changes in epidemiology, treatment, and survival over the last 20 years. Ann Surg 249: 63-71, 2009.

8. Overman MJ: Recent advances in the management of adenocarcinoma of the small intestine. Gastrointest Cancer Res 3: 90-96, 2009.

9. Sobin LH, Gospodarowicz MK and Wittekind C (eds): TNM Classification of Malignant Tumours. 7th edition. Wiley Blackwell, New York, pp153-159, 2009.

10. Aparicio T, Zaanan A, Svrcek M, Laurent-Puig P, Carrere N, Manfredi S, Locher C and Afchain P: Small bowel adenocarcinoma: Epidemiology, risk factors, diagnosis and treatment. Dig Liver Dis 46: 97-104, 2014.

11. Umman P, Adiyodi V and Narayan C: Small bowel adenocarcinoma - report of two cases and review of literature. Indian J Surg 75: 123-127, 2013.

12. Jovanovic I, Fry LC and Mönkemüller K: Small-bowel adenocarcinoma. Clin Gastroenterol Hepatol 9: A28, 2011.

13. Babba T, Schischmanoff O, Lagorce C, Wind P, Des Guetz G, Aparicio $T$ and Benamouzig R: Small bowel carcinoma revealing HNPCC syndrome. Gastroenterol Clin Biol 34: 325-328, 2010.

14. Aarnio M, Sankila R, Pukkala E, Salovaara R, Aaltonen LA, de la Chapelle A, Peltomäki P, Mecklin JP and Järvinen HJ: Cancer risk in mutation carriers of DNA mismatch-repair genes. Int J Cancer 81: 214-218, 1999.

15. Vasen HF, Wijnen JT, Menko FH, Kleibeuker JH, Taal BG, Griffioen G, Nagengast FM, Meijers-Heijboer EH, Bertario L, Varesco L, et al: Cancer risk in families with hereditary non polyposis colorectal cancer diagnosed by mutation analysis. Gastroenterology 110: 1020-1027, 1996.

16. Kunizaki M, Hidaka S, Isomoto H, Takeshita H, Nanashima A, Sawai T, Yasutake T and Nagayasu T: Diagnosis of small-bowel metastasis of hepatocellular carcinoma by double-balloon enteroscopy. Int J Surg Case Rep 3: 263-265, 2012.

17. Czaykowski P and Hui D: Chemotherapy in small bowel adenocarcinoma: 10-year experience of the British Columbia Cancer Agency. Clin Oncol (R Coll Radiol) 19: 143-149, 2007. 